\title{
A Goldbach Conjecture Using Twin Primes
}

\author{
By Dan Zwillinger
}

\begin{abstract}
The numbers $2 N=2(2) 1000000$ are checked to determine if they can be written as the sum of two twin primes. Thirty-three numbers are found that cannot be so represented; they are all less than 5000. The largest number in the range $2 N=$ $2(2) 500000$ that can be written as the sum of two twin primes in only one way is $2 N=24098$.
\end{abstract}

A natural extension of the Goldbach conjecture is to use only a restricted set of primes instead of all the primes. The primes used could be of a special form, or have special properties. This note describes the case where the allowed primes are twin primes $(3,5,7,11,13,17, \ldots)$.

Define $H(N)$ to be the number of decompositions of $N$ into two twin primes.

If a Goldbach type conjecture were to be true about twin primes, then the $H(N)$ function would have no zeros. Unfortunately, in the range $N=2(2) 500000, H(N)$ is equal to zero for the following values of $N$ :

$\begin{array}{llllll}94 & 96 & 98 & 400 & 402 & 404 \\ 514 & 516 & 518 & 784 & 786 & 788 \\ 904 & 906 & 908 & 1114 & 1116 & 1118 \\ 1144 & 1146 & 1148 & 1264 & 1266 & 1268 \\ 1354 & 1356 & 1358 & 3244 & 3246 & 3248 \\ 4204 & 4206 & 4208 & & & \end{array}$

A further computation found no additional zeros of $H(N)$ for $N$ in the range $500000(2) 1000000$. It is easy to show that if $H(6 N)=0$ then $H(6 N-2)=H(6 N+2)$ $=0$. This explains, somewhat, why the zeros of $H(N)$ come in threes.

Some interesting numbers concerning the $H(N)$ function: the smallest $N$ for which $H(N)=1000$ is $N=30240$, the largest $N$ such that $H(N)=1$ is $N=24098$.

This work was carried out on CCNY's computer system in early 1974.

Department of Applied Mathematics

California Institute of Technology

Pasadena, California 91125

Received February 23, 1978.

AMS (MOS) subject classifications (1970). Primary $10 \mathrm{~J} 15$. 